Voix et Images

voixetimages

\title{
De la compassion comme valeur romanesque
}

\section{Michel Biron}

Volume 31, numéro 1 (91), automne 2005

Figures et contre-figures de l'orientalisme

URI : https://id.erudit.org/iderudit/011931ar

DOI : https://doi.org/10.7202/011931ar

Aller au sommaire du numéro

\section{Éditeur(s)}

Université du Québec à Montréal

\section{ISSN}

0318-9201 (imprimé)

1705-933X (numérique)

Découvrir la revue

Citer ce compte rendu

Biron, M. (2005). Compte rendu de [De la compassion comme valeur romanesque]. Voix et Images, 31(1), 139-146. https://doi.org/10.7202/011931ar d'utilisation que vous pouvez consulter en ligne.

https://apropos.erudit.org/fr/usagers/politique-dutilisation/ 


\section{R O M A N}

De la compasion comme valeur romanesque

$++$

MICHEL BIRON

Université McGill

Dans son plus récent essai consacré au roman, Milan Kundera s'en prend aux amateurs de bons sentiments, à ceux qui voudraient que le roman, et à travers lui toute la littérature, les console des misères de l'humanité. Par exemple Sainte-Beuve écrivant ceci à propos de l'auteur de Madame Bovary: «Le reproche que je fais à son livre, c'est que le bien est trop absent ${ }^{1}$.» Le lecteur moderne ne peut que sourire devant une telle formule qui soumet la littérature à une exigence purement morale. Si ce lecteur a vécu sous le joug soviétique, comme c'est le cas de Kundera, il songera inévitablement aux ridicules prescriptions du réalisme socialiste, suivant lesquelles l'écrivain devait proposer une vision optimiste du monde, sans quoi il était considéré comme un auteur décadent et nuisible. Si ce lecteur a vécu au Québec, il songera aux non moins ridicules exhortations de l'Église appelant à une littérature saine et fortifiante.

Mais cela signifie-t-il que l'écrivain, pour être moderne, ou tout simplement pour être un véritable écrivain, doive absolument faire de l'ironie et se tenir à distance de toute morale? Ce n'est pas ce que fait Gustave Flaubert lorsqu'il répond à George Sand qu'il ne cherche pas à se moquer de ses personnages, mais veut au contraire «aller dans l'âme des choses ${ }^{2}$ ». Ce dont se moque avec raison Kundera, ce n'est pas la compassion qu'on peut éprouver à l'endroit de la pauvre Bovary (et que le roman de Flaubert exprime plus d'une fois); c'est de vouloir remplacer madame Bovary par des personnages autrement plus réjouissants qui reposeraient l'âme tourmentée du lecteur. La compassion constitue, elle aussi, une valeur romanesque. Non pas au même titre que l'ironie, qui est un procédé d'écriture, mais dans la mesure où elle permet justement, selon la formule de Flaubert, "d'aller dans l'âme des choses».

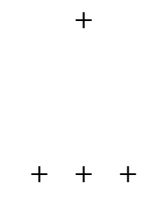

1 Sainte-Beuve, cité par Milan Kundera, Le rideau, Paris, Éditions Gallimard, 2005, p. 75.2 Gustave Flaubert, cité par Kundera, ibid., p. 76. 
Au Québec, Marie-Claire Blais appartient à cette catégorie d'écrivains mus par une infinie compassion à l'égard de personnages écorchés par la vie. Elle vient de terminer son roman le plus ambitieux, le dernier d'une trilogie commencée en 1995 avec Soifs ${ }^{3}$ et poursuivie en 2001 avec Dans la foudre et la lumière. Euvre foisonnante, difficile, incantatoire, cette trilogie de 800 pages comprend plusieurs dizaines de personnages, issus de tous les milieux (même si celui des artistes semble le mieux représenté) et qui se croisent dans une île au large du golfe du Mexique. Dans le dernier volet, Augustino et le chœur de la destruction, ils s'appellent Mère (Esther), Mélanie, Daniel, Mai, Samuel, Nora, Christiensen, Hans, Greta, Harriett, Charley, Marie-Sylvie, Vincent, Caroline, Jean-Mathieu, Olivier, Tchouan, Jermaine, Bernard, Valérie, Suzanne, Adrien, Charles, Frédéric, Cyril, Petites Cendres, la Vierge aux sacs, Franz, Arnie, Carlos, Lazaro, sans compter Marie Curie et bien sûr Augustino, qui est un enfant passionné par les livres et promis à une «haute destinée» (25). Au total, c'est plus de trente personnages qui se partagent la scène. Toute une société défile ainsi à un rythme fou, presque sans interruption. Comme dans les deux romans précédents, il n'y a aucune division par chapitre ou par paragraphe, les points sont extrêmement rares et les dialogues se fondent dans le récit sans autre indication que le changement des pronoms. Le langage ordinaire subit une accélération permanente ${ }^{4}$.

On finit par s'y reconnaître, grâce notamment à une série d'échos qui deviennent plus perceptibles encore dans ce troisième volume. Des noms de lieux par exemple, comme «rue Esmeralda, rue Bahama» à la sonorité magique qui ponctuait le deuxième volume reviennent au début d'Augustino comme pour faire transition. Chaque personnage est saisi à travers un motif principal qui réapparaît, lui aussi, tout au long du roman: la main tremblante de Mère, les inquiétudes maternelles de Mélanie, les missions humanitaires de Nora l'infirmière, la délicatesse orientale de Tchouan, la passion de la danse de Samuel, etc. Le procédé, plus musical que romanesque, produit un lyrisme envoûtant qui est encore plus évident si on lit le texte à haute voix.

Marie-Claire Blais renoue ainsi avec une écriture poétique déjà très présente dans ses premiers livres, notamment dans des récits comme Tête blanche ou Les voyageurs sacrés ${ }^{5}$. De tels textes, aujourd'hui oubliés, n'ont jamais fait le poids à côté d'Une saison dans la vie d'Emmanuel ${ }^{6}$ qui demeure, aux yeux de la critique, le grand livre de Marie-Claire Blais. Tout semble séparer ce roman aux phrases courtes

$$
+++
$$

3 Marie-Claire Blais, Soifs, Montréal, Boréal, 1995, 314 p. ; Dans la foudre et la lumière, Montréal, Boréal, 2001, 251 p.; Augustino et le chœur de la destruction, Montréal, Boréal, 2005, 302 p. 4 Il faudrait analyser en détail certains traits de l'écriture emportée de Marie-Claire Blais pour bien saisir en quoi elle dérange nos habitudes de lecture. C'est le cas en particulier des «et» disjonctifs, qui sont bien souvent les seuls signes d'un changement de scène. Plus perturbant encore, la valeur des virgules varie selon la place qu'elles occupent dans la phrase, comme on le voit dans l'exemple suivant où la troisième virgule a la valeur d'un point: « ce visage serait celui de Renata ou, plus tard, d'une autre femme, pour qui le connaissait, pensait Mère, il y avait toujours autour de Franz un parfum de scandale » (35). En remplaçant un point par une simple virgule, Marie-Claire Blais rapproche ce qui devrait être séparé et exige du lecteur qu'il ajuste le rythme de lecture non plus à partir des seules marques de ponctuation, mais aussi en fonction du contenu de la phrase. D'où la nécessité de revenir souvent en arrière pour corriger au fur et à mesure des "erreurs" possibles de lecture. 5 Id., Tête blanche, Québec, Institut littéraire du Québec, 1960; id., Les voyageurs sacrés, Montréal, HMH, 1969. 6 Id., Une saison dans la vie d'Emmanuel, Montréal, Éditions du Jour, 1965. 
et à l'ironie sèche du souffle poétique et de la compassion qui caractérisent ses romans ultérieurs, depuis Le sourd dans la ville et Visions d'Anna ${ }^{7}$ jusqu'à cette trilogie qui constitue toutefois moins une seconde manière que l'aboutissement de toute son œuvre. Car l'œuvre de Marie-Claire Blais possède une réelle cohérence, qui se révèle aujourd'hui mieux que jamais, un peu comme celle de Gabrielle Roy qu'on a appris à relire à partir de livres plus intimistes, longtemps relégués au second plan derrière le chef-d'œuvre réaliste qu'a été Bonheur d'occasion. Il n'est pas excessif de penser, en lisant la trilogie, que le centre de gravité de l'œuvre de Marie-Claire Blais vient de se déplacer radicalement. Du moins, on ne pourra plus continuer à considérer cette production uniquement à partir d'Une saison dans la vie d'Emmanuel. D'ailleurs, ce roman au style si serré est-il si différent du lyrisme grandiose d'Augustino? La césure entre les deux esthétiques, entre la révolte d'Une saison dans la vie d'Emmanuel et le mélange de colère et de pitié qui anime toute la trilogie, ne doit pas être exagérée (elle n'a rien de comparable avec celle qui sépare par exemple l'expérience ratée d'Un joualonais, sa Joualonie ${ }^{8}$ du reste de l'œuvre). Il suffit de relire la première page d'Une saison dans la vie d'Emmanuel pour trouver une longue description (des pieds de Grand-Mère Antoinette) qui aurait très bien pu se retrouver telle quelle dans Augustino. De même pour le couple de Jean Le Maigre et de Grand-Mère Antoinette auquel il est tentant de comparer celui d'Augustino et de sa grand-mère. Plus généralement, c'est toute l'œuvre de Marie-Claire Blais qui est traversée par la thématique de la souffrance et du mal.

Dans la trilogie, les images d'apocalypse se succèdent d'un bout à l'autre, comme la pulsation d'un monde livré à une sorte de terreur sacrée. Ce sont par exemple les portraits de kamikazes, "ces nouveaux anges de la mort» (17), qui rappellent à Lazaro la violence de son Égypte natale. Ou les images d'une centaine de dauphins venus s'échouer sur une plage du Venezuela et qui se retrouvent sur l'écran de l'ordinateur d'Augustino. Images insensées d'un monde où la nature elle-même apparaît déréglée. Mère ne trouve aucune explication rationnelle à ce qu'elle voit et demande à Augustino d'éteindre son ordinateur. La séquence ne dure qu'un moment, mais elle est typique du roman, qui se construit ainsi par petits plans juxtaposés, comme autant de fragments d'un désastre, comme autant de voix qui forment, malgré elles, «le chœur de la destruction». Les personnages eux-mêmes semblent frappés de stupeur, comme en marge de leur propre vie, immobiles au cœur d'un monde suragité, prêts à s'évanouir dans le néant. On a l'impression de voir au ralenti des anges et des ombres qui chutent avec, en arrière-plan, un flot d'images juxtaposées.

Dans l'une de ses notes américaines publiées il y a une dizaine d'années, Marie-Claire Blais se souvenait d'un reproche que lui avait fait le grand critique Edmund Wilson: «Il faut que ce soit plus serré, pensez à la poésie de Rimbaud, il est jeune, mais c'est très serré. Resserrez davantage, le flot n'est pas assez maintenu, dirigé 9 .» Elle ajoutait alors: «Edmund et moi n'entendions pas la même musique.»

7 Id., Le sourd dans la ville, Montréal, Stanké, 1979; id., Visions d'Anna, Montréal, Stanké, 1982.8 Id., Un joualonnais, sa Joualonnie, Montréal, Éditions du Jour, 1973. 9 Id., Parcours d'un écrivain. Notes américaines, Montréal, VLB éditeur, 1993, p. 52. 
C'est que la sienne, précisait-elle encore, passe moins par Rimbaud que par la révolution féministe. Un tel aveu a quelque chose de compromettant, comme si la littérature s'avouait seconde derrière le combat idéologique. Et on peut penser que certains livres de Marie-Claire Blais pêchent justement par là. On peut même penser que c'est encore le cas dans cette trilogie, dont le registre apocalyptique est racheté par une vision du monde écologiste et humanitaire. Ainsi, par exemple, de Nora, infirmière en Afrique, et qui éprouve une compassion infinie pour les malheurs d'autrui. Elle envie son ami philosophe, Bernard, capable de mettre des limites raisonnables à sa générosité ( on ne peut être responsable de tout ce qui arrive» [207], répète-t-il). Les femmes n'ont pas ce talent, explique Mère, qui prend aussitôt la défense de l'épouse de Bernard, Valérie. Celle-ci écrit des romans d'essence philosophique, mais elle subit sans cesse le mépris des collègues masculins de Bernard qui jugent impossible qu'une femme puisse être véritablement philosophe. Comme Nora ou Mère, mais aussi comme la plupart des personnages féminins, Valérie se réclame d'une vision humanitaire du monde ( son humanité était sa science et le domaine de ses interrogations et réflexions» [208]). On retrouve ici les préoccupations féministes de Marie-Claire Blais, celles qui la séparaient si radicalement de la soi-disant vanité masculine de son maître Edmund Wilson.

Mais ce n'est pas ce qu'on retient au moment où on termine la lecture de la trilogie. C'est plutôt la musique du texte, la maîtrise du flot, de même que la finesse des détails, l'extraordinaire sensibilité des portraits de chacun des personnages et la solidité du tableau final. La vérité des personnages ne vient pas de leur pertinence sociologique ou morale, avec laquelle on peut être ou non d'accord, mais de la vie ellemême. Leurs voix entrecroisées sont appelées par la composition romanesque elle-même et s'intègrent les unes aux autres dans une construction polyphonique qui a sa beauté, sa grandeur.

À l'inverse de l'écriture emportée de Marie-Claire Blais, le court récit autobiographique que Francine Noël vient de publier, La femme de ma vie ${ }^{10}$, est une œuvre de retenue, centrée sur la figure de la mère de l'écrivaine. La surprise est d'autant plus grande que l'auteure nous a habitués, depuis Maryse ${ }^{11}$, à de vastes tableaux de société. Mais la continuité entre les deux livres, écrits l'un et l'autre dans une langue alerte et agréable, est évidente et l'on comprend mieux, lisant La femme de ma vie, à quel point Maryse était déjà une œuvre en partie autobiographique, un roman familial. Du reste, La femme de ma vie ne se limite pas au seul portrait maternel; le récit constitue aussi, quoique sur un ton plus intimiste, un passionnant tableau de société, avec Montréal en arrière-plan. D'origine modeste, célibataire dès la fin des années 1950, c'est-à-dire à une époque où on ne l'était guère, la mère de Francine Noël vient de Québec et du Bas-du-Fleuve, mais s'intègre à Montréal au milieu d'im-

$$
++
$$

10 Francine Noël, La femme de ma vie, Montréal, Leméac, 2005, 165 p. teur, 1983, $432 \mathrm{p}$.

11 Id., Maryse, Montréal, VLB édi- 
migrants venus de partout. Elle est parfaitement à l'aise dans cet univers multiculturel avant la lettre.

Cette mère fait aussi un excellent personnage romanesque, original à souhait. En avance sur son temps, elle exerce quantité de métiers (de vendeuse de produits cosmétiques à secrétaire légale), voulant à tout prix montrer à sa fille universitaire qu'elle ne vivrait jamais à son crochet. Elle se compose une culture étonnante, volontiers théâtrale, faite en partie pour épater la galerie, mais moins par snobisme que par gaieté enfantine. Elle connaît par cœur les Fridolinades de Gratien Gélinas, se nourrit d'histoires tirées de la mythologie grecque ou de la Bible et suit des cours de diction en récitant des poèmes de Sully Prudhomme. Plus elle vieillit, plus cette mère devient une femme excentrique. Elle finit sa vie dans les Rose-Croix tout en se passionnant pour la spéléologie et la minéralogie. De toutes ses qualités, celle à laquelle Francine Noël est la plus sensible est son art de raconter. Elle lui reconnaît surtout «la grâce de la relation" (27). Le mot "relation», dans ce contexte, n'a qu'un sens: c'est celui de récit. Ici, ce n'est pas seulement la fille qui parle, c'est aussi la romancière.

Le récit insiste, comme on peut s'y attendre, sur les difficiles relations mèrefille. Femme orgueilleuse, la mère ne pardonne pas à sa fille de l'avoir quittée à dixsept ans. Les scènes de rupture se multiplient jusqu'à la fin, sous d'autres prétextes que Francine Noël ne cherche pas à expliciter. Il ne s'agit pas d'un règlement de comptes, mais d'un livre «mémorial» (165). En cela, il s'apparente à nombre de récits contemporains, appelés par Dominique Viart des récits de filiation ${ }^{12}$. L'accent n'est pas mis directement sur soi-même, comme dans une autobiographie traditionnelle, mais sur un personnage autre qui, si familier soit-il, si déterminant soit-il pour l'identité de la narratrice, demeure une sorte d'énigme. Le statut de la mère reste en effet ambigu, mi-réel et mi-fictif. Les souvenirs ne suffisent d'ailleurs pas à reconstituer la vie de ce personnage secret, qui échappe en grande partie au récit de sa fille. Celleci se surprend à découvrir de nombreux trous, notamment la vie amoureuse de sa mère, dont elle ignore à peu près tout. L'héritage familial qu'elle incarne n'est pas un poids dont il lui faut se défaire pour s'épanouir, mais plutôt un legs imprécis auquel elle finit par consentir, à la fois pour rendre hommage à «la femme de sa vie» et pour lutter «contre l'envasement de la mort» (165). C'est aussi un exercice littéraire particulièrement délicat et réussi.

Nikolski, premier roman de Nicolas Dickner ${ }^{13}$, a reçu un accueil critique enthousiaste dès sa sortie au début de 2005. Avec raison, car il s'agit d'un roman porté par une allégresse et une sorte de délire imaginatif qui ne sont pas courants dans la

$$
+++
$$

12 Dominique Viart, "Filiations littéraires», Jan Baetens et Dominique Viart (dir.), Écritures contemporaines, t. II. États du roman contemporain. Actes du colloque de Calaceite 6-13 juillet 1996, Paris-Caen, Minard, coll. «Lettres modernes», 1999, p. 115-131. 13 Nicolas Dickner, Nikolski, Québec, Nota bene, coll. «Alto», 2005, $326 \mathrm{p}$. 
littérature actuelle, où dominent les mille et une espèces d'écritures de soi. Ici, les fenêtres du monde s'ouvrent toutes grandes, et des personnages à moitié déjantés plongent dans l'époque actuelle à la recherche de leur origine. Ils vivent des «aventures merveilleuses" qui n'en sont pas, entourés d'animaux faussement mythiques et d'ordinateurs qui sont déjà plus vieux qu'eux et qui ont même l'air franchement archaïques. Avec un principe à la clef: «Tout est déchet.» (135) S'il est un champ lexical qui définit ce roman, c'est bien celui des vidanges et des débris en tous genres, qui sont les traces les plus sûres de ce qu'est vraiment une communauté: «Les déchets dévoilent ce que tout le reste tente de cacher. » (150)

Le roman se construit autour de trois personnages principaux, Noah, Joyce et un narrateur qui commence par dire: «Mon nom n'a pas d'importance.» (9) Les récits s'entrecroisent de la façon apparemment aléatoire, mais se ressemblent tous selon un principe de symétrie suggéré par un mystérieux livre à trois têtes qui se retrouve dans la librairie où travaille le narrateur. Parmi les objets-fétiches qui décident du sort des personnages se trouve un compas baptisé le compas Nikolski. Cet objet apparaît au début du roman alors que le narrateur est en train de remplir trente sacs de vidange (c'est-à-dire «mille huit cents litres d'ultra-plastique» [25]) et de classer les biens laissés par sa mère qui vient de mourir. Ce compas de marine est censé indiquer le nord; or, et c'est là tout le problème, il est légèrement défectueux et pointe à gauche du nord, en direction d'un minuscule village, appelé Nikolski, situé au large de l'Alaska. C'est aussi de là que Noah a reçu la dernière carte postale de son père, Jonas Doucet, lequel se révèle être aussi celui de Joyce. Il est nomade, comme la mère de Noah qui habite une roulotte qu'elle déplace à travers les prairies canadiennes. Pour écrire à celle-ci, Noah doit calculer ses déplacements et envoyer sa lettre en "poste restante». Ses lettres, de toute façon, lui sont systématiquement retournées, comme celles qu'il adresse à son père.

À dix-sept ans, Noah s'en va étudier à Montréal, où il apprend le surprenant métier d'archéologue postindustriel. Il habite la Petite Italie, à deux pas de l'appartement loué par Joyce, qui vend des poissons le jour et fait du piratage informatique la nuit. Ils ne se rencontrent pas. Avec humour, ce roman s'amuse ainsi à créer des attentes qu'il s'applique ensuite à décevoir, comme pour échapper aux conventions de la fiction. On retrouve Noah un peu plus tard au Venezuela où il passe quelques années à s'occuper d'un enfant qui est peut-être le sien et qu'il ramène ensuite à Montréal. À l'aéroport, il croise Joyce, mais la rencontre imaginée par le lecteur n'a toujours pas lieu. Joyce s'en va on ne sait où, munie de guides de voyage «empruntés " au narrateur. Le roman se termine sans qu'ils se soient connus, chacun retournant à sa vie de déraciné. Le voyage au bout de cette Amérique déboussolée s'achève, comme il se doit, dans la librairie du narrateur, où Noah achète toute une pile de livres sur les dinosaures pour son fils putatif.

L'écriture de Nicolas Dickner rappelle, par bien des côtés, celle de Jacques Poulin: phrases simples, paragraphes le plus souvent courts, dialogues épurés, expressions typiquement pouliniennes (comme le verbe "emprunter» utilisé au sens de «voler» ou l'expression "on a l'air d'une bande de zouaves»), fréquentes énumérations, accumulation de petites histoires, de digressions, recours à des genres anciens comme l'épopée ou la légende, précision maniaque de certains détails, 
intégration de formes non littéraires (comme les instructions, les annonces classées, les descriptions scientifiques, les expressions aperçues sur des pancartes extérieures, etc.), jeux de miroir, références abondantes au monde du livre et de l'écriture (librairie, noms d'écrivains, titres de livres, etc.), divers procédés autoréflexifs qui mettent la réalité à distance, refus de se prendre au sérieux, banalité du décor, expérience de l'errance, etc. Nikolski est une sorte de Volkswagen Blues des années 2000, une traversée immobile de l'Amérique.

Mais cette imitation n'enlève rien à la singularité du roman de Nicolas Dickner, qui fait du mimétisme une sorte de postulat général, à la fois d'ordre moral et esthétique. Nikolski, en outre, se laisse envahir par tout ce qui l'environne, peu importe qu'il s'agisse de fiction ou de réalité. Il s'agit de coller au monde des signes, mais en même temps d'ouvrir ce monde à des univers de sens inattendus, notamment par le télescopage du passé et du présent. La description des objets et des lieux devient une aventure en soi, un voyage dans le temps et dans l'espace. Tout fait signe, tout est soluble dans l'écriture romanesque. Du compas Nikolski au dépotoir d'ordinateurs découvert par Joyce, le roman est une collection de choses hétéroclites, de curiosités postmodernes ou de vieilleries inutiles qui deviennent les emblèmes d'un monde surchargé où rien d'original n'existe, où tout est indéfiniment recyclable, même les histoires les plus éculées, où le meilleur moyen de survivre est de passer inaperçu, de se fondre dans le décor. Voici par exemple le poisson préféré de Joyce, la plie:

Ce pleuronectidé peu glorieux, ni redoutable ni athlétique, maîtrise le mimétisme au plus haut degré. Sa silhouette aplatie et une complexe pigmentation de l'épiderme lui permettent de se confondre totalement avec le fond marin. Immobile, elle disparaît; en mouvement, elle ressemble à un simple nuage de sable poussé par le courant (193).

Par analogie, on comprend bien sûr que Joyce se reconnaît parfaitement dans cet art du camouflage, comme lorsqu'elle s'habille tout en noir pour ne pas être vue, la nuit, en train de fouiller les conteneurs emplis de débris d'ordinateurs. C'est cet art qui importe ici, bien plus que l'action elle-même. Le roman invente un univers à la fois baroque et familier, où les correspondances entre la réalité quotidienne et une sorte de métaphysique du déchet se multiplient à l'infini. Roman étonnant, vif et plein d'humour, Nikolski jette sur le monde actuel un regard à la fois désespéré et serein. Il n'y a ici ni révolte ni bon sentiment, mais une manière toute personnelle d'habiter un monde où même les compas semblent avoir perdu le nord.

Si différents soient-ils, ces trois romans ont en commun de peindre avec chaleur, sur un ton tantôt grave, tantôt léger, des personnages désorientés à la recherche de leur famille, et en particulier de leur mère. Le personnage de Mère, chez Marie-Claire Blais, est central, tout comme la mère de Francine Noël et même celle de Noah dans Nikolski, errant au milieu des prairies canadiennes. L'individu aujourd'hui semble si 
peu sûr du lien social et de son origine qu'il tente de tout reconstruire et de se refaire une généalogie. Pas question de creuser la distance qui sépare cet individu de sa famille ou de sa société, puisque cet individu souffre déjà d'un excès de distance. «Aller au fond des choses», dans cette perspective, c'est pénétrer au cœur de cette distance, c'est ressentir concrètement le vertige de l'individu contemporain. Non pas pour réparer naïvement les choses, mais parce que c'est ainsi qu'il vit. 